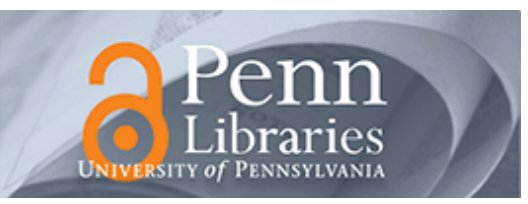

University of Pennsylvania

ScholarlyCommons

July 2001

\title{
Computational Modeling of Medium Spiny Projection Neurons in Nucleus Accumbens: Toward the Cellular Mechanisms of Afferent Stream Integration
}

\author{
John A. Wolf \\ University of Pennsylvania \\ Lee F. Schroeder \\ University of Pennsylvania \\ Leif H. Finkel \\ University of Pennsylvania, leif@neuroengineering.upenn.edu
}

Follow this and additional works at: https://repository.upenn.edu/be_papers

\section{Recommended Citation}

Wolf, J. A., Schroeder, L. F., \& Finkel, L. H. (2001). Computational Modeling of Medium Spiny Projection Neurons in Nucleus Accumbens: Toward the Cellular Mechanisms of Afferent Stream Integration. Retrieved from https://repository.upenn.edu/be_papers/10

Copyright 2001 IEEE. Reprinted from Proceedings of the IEEE, Volume 89, Issue 7, July 2001, pages 1083-1092.

Publisher URL: http://ieeexplore.ieee.org/xpl/tocresult.jsp?isNumber=20336\&puNumber=5

This material is posted here with permission of the IEEE. Such permission of the IEEE does not in any way imply IEEE endorsement of any of the University of Pennsylvania's products or services. Internal or personal use of this material is permitted. However, permission to reprint/republish this material for advertising or promotional purposes or for creating new collective works for resale or redistribution must be obtained from the IEEE by writing to pubs-permissions@ieee.org. By choosing to view this document, you agree to all provisions of the copyright laws protecting it.

This paper is posted at ScholarlyCommons. https://repository.upenn.edu/be_papers/10

For more information, please contact repository@pobox.upenn.edu. 


\title{
Computational Modeling of Medium Spiny Projection Neurons in Nucleus Accumbens: Toward the Cellular Mechanisms of Afferent Stream Integration
}

\author{
Abstract \\ The nucleus accumbens (Nacc) regulates the major feedback pathways linking prefrontal cortex, \\ hippocampus, and amygdala. We describe simulations of a biophysical level model of a single medium \\ spiny projection (MSP) neuron, the principle cell of the Nacc. The model suggests that the unusual \\ bistable membrane potential of MSP cells arises from the interplay between two potassium currents, $\mathrm{K}_{\mathrm{IR}}$ \\ and $\mathrm{K}_{\mathrm{A}}$. We find that the transition from the membrane potential down state $(\sim-85 \mathrm{mV})$ to the upstate \\ $(\sim-60 \mathrm{mV})$ requires a significant barrage of synchronized inputs, and that ongoing afferent stimulation is \\ required to maintain the cell in the up state. The Nacc receives the densest dopaminergic innervation in \\ the brain, and the model demonstrates, in agreement with recent experimental evidence, that dopamine \\ acts to increase the energy barrier to membrane potential state transitions. Through its action on $\mathrm{K}_{\mathrm{IR}}$ and \\ L-type $\mathrm{Ca}^{2+}$ channels, dopamine selectively lowers cell gain in the down state and increases it in the up \\ state, a mechanism for context-dependent gain control. \\ These findings suggest a mechanism of afferent pattern integration in the accumbens arising from \\ transient synchronization among ensembles of MSP neurons. We attempt to relate these findings to \\ possible origins of abnormalities of sensory gating in schizophrenia.

\section{Keywords} \\ Bistability, frontal cortex, medium spiny projection (MSP) neuron, modeling, simulation, striatum.

\section{Comments} \\ Copyright 2001 IEEE. Reprinted from Proceedings of the IEEE, Volume 89, Issue 7, July 2001, pages \\ 1083-1092. \\ Publisher URL: http://ieeexplore.ieee.org/xpl/tocresult.jsp?isNumber=20336\&puNumber=5 \\ This material is posted here with permission of the IEEE. Such permission of the IEEE does not in any way \\ imply IEEE endorsement of any of the University of Pennsylvania's products or services. Internal or \\ personal use of this material is permitted. However, permission to reprint/republish this material for \\ advertising or promotional purposes or for creating new collective works for resale or redistribution must \\ be obtained from the IEEE by writing to pubs-permissions@ieee.org. By choosing to view this document, \\ you agree to all provisions of the copyright laws protecting it.
}




\title{
Computational Modeling of Medium Spiny Projection Neurons in Nucleus Accumbens: Toward the Cellular Mechanisms of Afferent Stream Integration
}

\author{
JOHN A. WOLF, LEE F. SCHROEDER, AND LEIF H. FINKEL
}

Invited Paper

The nucleus accumbens (Nacc) regulates the major feedback pathways linking prefrontal cortex, hippocampus, and amygdala. We describe simulations of a biophysical level model of a single medium spiny projection (MSP) neuron, the principle cell of the Nacc. The model suggests that the unusual bistable membrane potential of MSP cells arises from the interplay between two potassium currents, $K_{\mathrm{IR}}$ and $K_{\mathrm{A}}$. We find that the transition from the membrane potential down state $(\sim-85 \mathrm{mV})$ to the upstate $(\sim-60$ $m V)$ requires a significant barrage of synchronized inputs, and that ongoing afferent stimulation is required to maintain the cell in the up state. The Nacc receives the densest dopaminergic innervation in the brain, and the model demonstrates, in agreement with recent experimental evidence, that dopamine acts to increase the energy barrier to membrane potential state transitions. Through its action on $K_{\mathrm{IR}}$ and L-type $\mathrm{Ca}^{2+}$ channels, dopamine selectively lowers cell gain in the down state and increases it in the up state, a mechanism for context-dependent gain control.

These findings suggest a mechanism of afferent pattern integration in the accumbens arising from transient synchronization among ensembles of MSP neurons. We attempt to relate these findings to possible origins of abnormalities of sensory gating in schizophrenia.

Keywords-Bistability, frontal cortex, medium spiny projection (MSP) neuron, modeling, simulation, striatum.

\section{INTRODUCTION}

Consider the following question: to what degree is it possible to develop a biophysical-level, computational model of

Manuscript received December 1, 2000; revised March 1, 2001. This work was supported by the National Science Foundation under Grant ECS98-73463, by the David and Lucile Packard Foundation, and by the Office of Naval Research.

The authors are with the Department of Bioengineering and Institute of Neurological Sciences, University of Pennsylvania, Philadelphia, PA 19104-6392 USA (e-mail: leif@neuroengineering.upenn.edu; schroed@ neuroengineering.upenn.edu; wolfjo@mail.med.upenn.edu).

Publisher Item Identifier S 0018-9219(01)05409-3. schizophrenia? Given that the computational principles of well-characterized neural structures such as the retina or the stomatogastric ganglia are not completely understood, what hope is there of modeling a complex disorder that involves pathologic changes in multiple brain areas, disorders of neuromodulation, complicated genetic expression, and protean behavioral manifestations?

On the other hand, complex pathologies at the receptor, cellular, and behavioral levels might all conceivably be manifestations of a singular perturbation at the computational level—much as a change in economic system (e.g., socialism to capitalism) is associated with changes on many levels of society, from a rise in skiing injuries to genetically modified corn proteins.

In this paper, we will consider the hypothesis that the nucleus accumbens (Nacc), an integrative center located in the subcortical basal forebrain, may be the epicenter of dysfunction in schizophrenia. The evidence for pathologic changes in Nacc in schizophrenic brains is controversial [1]-[3]. However, Nacc sits at and regulates the crossroads of the major psychomotor pathways of the brain, receiving major afferent projections from prefrontal cortex, hippocampus, and amygdala. Each of these projecting areas exhibits gross pathologic changes in schizophrenic brains, thereby affecting the process of integration carried out in Nacc. Fig. 1 shows the anatomical connections of the Nacc.

Several features of Nacc make it a fascinating structure to model in its own right. First, the nucleus is composed almost entirely of inhibitory cells; medium spiny projection (MSP) neurons, the principle cells of the nucleus, comprise $\sim 95 \%$ of the cells. MSP neurons are densely interconnected both by gap junctions and $\mathrm{GABA}_{\mathrm{A}}$ synapses. The MSP cells display an unusual "bistable" two-state membrane potential. In the "down" state (resting potential $\sim-85 \mathrm{mV}$ ) cells are restricted 


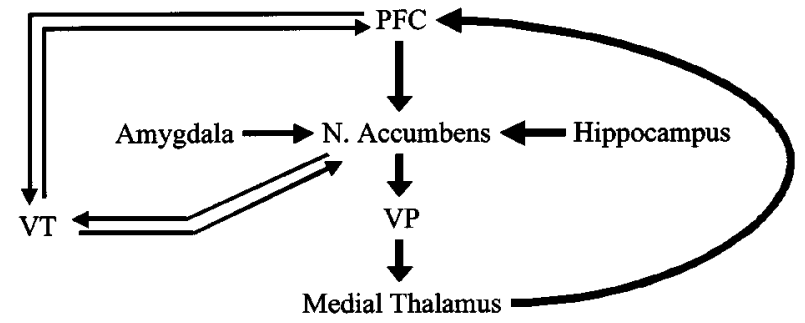

Fig. 1. Anatomical connections of the Nacc. The accumbens is the major subcortical projection site of prefrontal cortex, and projects back to PFC via the ventral pallidum (VP). PFC and N. accumbens together control the activity of the ventral tegmentum (VT) and substantia nigra pars compacta - the major dopaminergic nuclei which project densely back to both areas. N. accumbens also receives major projections from hippocampus and amygdala Output from VP also regulates the thalamic reticular nucleus, which mediates cortico-thalamic interactions (not shown).

from firing; in the "up" state (resting potential $\sim-60 \mathrm{mV}$ ) cells can be depolarized into firing [4]-[6]. State transitions occur in a coordinated manner among coupled MSP neurons in local anatomical microzones [7].

The physiological properties of MSP cells, and their ability to switch between states, are modulated by dopamine. The accumbens receives a massive dopaminergic input from the ventral tegmentum, and together with prefrontal cortex, Nacc controls the release of dopamine by ventral tegmentum. ${ }^{1}$ At the cellular level, dopamine modulates several intrinsic currents, including a persistent $\mathrm{Na}^{+}$channel, L-type $\mathrm{Ca}^{2+}$ channels and an anomalous inward rectifying potassium current, $\mathrm{K}_{\mathrm{IR}}$. Malenka et al. [11] marshal evidence that dopamine acts to stabilize MSP neurons in either the up or down states by effectively increasing the energy barrier to state transitions.

Grace et al. [12] have put forward an intriguing theory in which these transitions between the "up" and "down" states allow MSP neurons to act as a gate controlling information flow along the reentrant loop linking prefrontal cortex, ventral striatum and thalamus. In the up state, the gate is open, and in the down state, it is closed. They propose that input from the hippocampus opens the gate by depolarizing MSP neurons into the up state. Experimental evidence shows that stimulation of the hippocampus induces a long-lasting transition to the up state, and transection of the hippocampal fornix, or injection of the anesthetic lidocaine into the fornix, suppresses the up state [13]. Grace et al. account for the major symptoms of schizophrenia: disorganized thinking, impulsive behavior, delusions, hallucinations, perseverative behavior, and psychomotor retardation as resulting from a number of specific effects of abnormal dopaminergic modulation.

The metaphor of "gating" ties into a large body of research linking schizophrenia to defects in sensorimotor gating. In-

\footnotetext{
${ }^{1}$ The release of dopamine is tied to reward and addictive behavior, and shows pervasive abnormalities in schizophrenic brains. Weinberger [8] put forward an influential hypothesis that in schizophrenia, hypoactivation of PFC leads to disinhibition of ventral tegmentum and consequently increased dopaminergic activation of Nacc. Recent experimental evidence supports this hypothesis [9], [10].
}

adequate filtering of incoming stimuli and inaccurate coupling to motor response are posited to lead to the behavioral abnormalities. One paradigm commonly used to assess sensorimotor gating is prepulse inhibition (PPI) of the acoustic startle reflex. In this paradigm, a short, soft auditory tone suppresses the startle response elicited by a loud tone blast-when the prepulse tone precedes the blast by a fraction of a second. In humans, the startle response consists of eye closure and tightening of facial and neck muscles - other species exhibit more dramatic responses, e.g., the armadillo jumps several feet straight up in the air. More to the point, schizophrenic subjects show diminished PPI, which is interpreted as a sign of abnormal sensory gating. PPI is mediated by the pedunculopontine tegmental nucleus, which is controlled by the Nacc via its projection to the pontine reticular formation [14]. Diseases affecting the dorsal striatum, such as Huntington's disease and Tourette's syndrome, also show abnormal PPI. ${ }^{2}$ Swerdlow et al. [15] have shown that increased dopaminergic activity disrupts PPI via activation of dopamine (D2) receptors on MSP cells. These dopamine-induced PPI deficits are ameliorated by haloperidol and other antipsychotic agents.

It is attractive to consider the two-state membrane potential of the MSP neuron as a gating mechanism, but other interpretations are possible. Consider, for example, the somewhat subtle difference between "gating" and "routing." In a telephone switching network, the pulsed input of the dialed phone number determines which of multiple possible output routes is opened. This is gating, but with multiple possible gates, only one of which is opened-and the afferent data stream provides the information specifying which gate is the correct one. Might the pattern of hippocampal input to accumbens determine how the prefrontal cortical input is coupled back to PFC, or equivalently, the pattern of activity to be reentered to PFC? Other recent experimental data suggests that transitions between MSP bistable states can be driven by the PFC input itself [16]. Thus, the accumbal networks might be envisioned as carrying out some form of computation on the combined inputs from PFC, hippocampus, amygdala $^{3}$ and elsewhere, with the result of this computation determining the information content of the accumbal output as well as its neuronal targets. In such a mechanism, the accumbens would fuse multiple afferent information streams: the PFC providing goal-directed information and the hippocampus providing current environmental context. In other words, the accumbens integrates the "will" and the "way."

In line with this interpretation, Lopes da Silva et al. have proposed that "behaviorally meaningful information in the $\mathrm{Nacc}$ is represented by fine-grained spatiotemporal firing patterns" of local ensembles of MSP neurons [16]. Thus, the local firing pattern represents a behaviorally meaningful

\footnotetext{
${ }^{2} \mathrm{Nacc}$ is composed of a shell and core regions. Anatomically, the shell is an extension of the amygdala and the core is contiguous with the dorsal striatum (caudate/putamen). However, MSP neuron morphologies are similar in both regions, as are the electrophysiological characteristics of the cells [16].

${ }^{3}$ The amygdala is thought to provide affective (emotion-associated) contextual information.
} 
integration of the multiple inputs to accumbens, and the information is encoded in the spatiotemporal pattern of MSP cell activity.

As a first step toward understanding these issues, we wish to examine the cellular mechanisms operating in MSP neurons. In particular, how do the specific afferent spike trains impingent upon the network determine transitions between states and the actual firing of MSP neurons. We are motivated by several mechanistic questions.

- What is the biophysical mechanism underlying the bistable membrane potential of MSP neurons?

- What determines transitions between membrane states-i.e., are there spatial and/or temporal requirements on the afferent spike trains?

- Can a model disambiguate the role of PFC versus hippocampal inputs regarding state transitions and MSP firing?

- What types of computations might be carried out by such a network?

Lopes da Silva et al. provide a motivation for these questions in their comprehensive review [16]. Making best-guess approximations for membrane resistances and single-afferent postsynaptic currents, they calculate that $15-50$ input spikes would be required to switch cells from the down to the up state. They rued however, that this back-of-the-envelope calculation could not take account of effects due to the $\mathrm{K}_{\mathrm{IR}}$ current, dendritic geometry, and other complicating factors. As Wilson [4] has further pointed out, in the down state, the large open $\mathrm{K}_{\mathrm{IR}}$ conductance reduces the membrane time constant, and thus the synaptic integration time. All of the received inputs might have to occur within a narrow time window $(\sim 2-5 \mathrm{~ms})$ in order to kick the cell into the up state. Once in the up state, closing of the voltage-dependent $\mathrm{K}_{\mathrm{IR}}$ channel would increase the membrane time constant, allowing inputs received over longer time windows to maintain the depolarized state or induce firing.

As a first objective, we thus sought to develop a biophysical-level model of a single MSP neuron with which one could investigate the number of inputs required to switch cell state, and the effect of dopamine on this process.

\section{RESULTS}

\section{A. Constructing a Model MSP Neuron}

We constructed a reduced model of an MSP neuron using GENESIS [17]. In vivo, MSP neurons have small to medium somata $(15.8 \pm 2.8 \mu \mathrm{m})$ and a multipolar or fusiform shape from which 3-6 primary dendrites arise (diameter, 1-2 $\mu \mathrm{m}$ ). The first dendritic branch point arises 20-30 $\mu \mathrm{m}$ out from the soma, and the secondary and higher order dendrites are densely covered with spines (the primary dendrites are smooth) [16]. MSP neurons in the core and shell regions of Nacc, have largely similar morphological and physiological properties [18] and their differences have been neglected here. The cells are electrotonically compact, with an input resistance measured at $217 \pm 33 \mathrm{M} \Omega$ in whole cell patch clamp [16]. These properties are captured in a
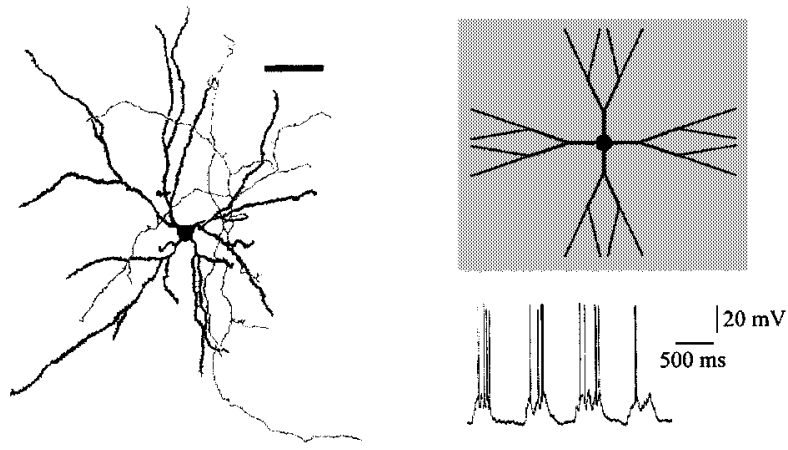

Fig. 2. Medium spiny projection (MSP) neuron. A stained MSP neuron, the principle cell of the Nacc, is shown on the left [23]. (Top right) Schematic of the 29-compartment cell model used in the simulations. (Bottom right) Recording from MSP neuron showing the up and down bistable membrane potential states, with action potentials fired from the up state. (From O'Donnell et al. [49], time interval shown is $2.5 \mathrm{~s}$.)

29-compartment model, with four primary dendrites, shown in Fig. 2.

MSP cells contain a number of active currents, including voltage-dependent $\mathrm{Na}^{+}$and delayed rectifier channels, a persistent $\mathrm{Na}^{+}$current, a variety of $\mathrm{Ca}^{2+}$ channels ( $\mathrm{L}, \mathrm{N}, \mathrm{P} / \mathrm{Q}$, $\mathrm{R}$, and $\mathrm{T}$ ), and a $\mathrm{K}_{\mathrm{A}}$ current. Most interestingly, cells contain an unusual, inward-rectifying $\mathrm{K}^{+}$channel $\left(\mathrm{K}_{\mathrm{IR}}\right)$ that is active at hyperpolarized potentials [6]. The $\mathrm{K}_{\mathrm{IR}}$ channel underlies the unusually hyperpolarized "down" state $(\sim-85 \mathrm{mV})$. This channel has extremely fast kinetics, and does not inactivate-rather, it closes with minimal depolarization, effectively shutting off by $-65 \mathrm{mV}$. The $\mathrm{K}_{\mathrm{IR}}$ current flows outward (hyperpolarizing) for all voltages above the reversal potential $(-90 \mathrm{mV})$; the channel is called an "inward rectifier" because it preferentially allows inward current at membrane potentials more negative than $-90 \mathrm{mV}$.

There is no direct data on $\mathrm{K}_{\mathrm{IR}}$ kinetics in accumbal cells, and so we adapted Hodgkin-Huxley $\alpha$ and $\beta$ parameters from Hayashi and Freeman's studies of $\mathrm{K}_{\mathrm{IR}}$ in Aplysia [19], and modified these parameters until they generated a current-voltage $(I-V)$ curve that matched that published by Wilson [5]. Wilson generated his $I-V$ curve from Hagiwara and Takahasi's data on $K_{I R}$ in starfish eggs [20], together with membrane resistances as determined in spiny neostriatal cells. Our resulting $I-V$ curve is shown in Fig. 3. Clearly, extrapolating from invertebrates, compensating for temperature differences, and other factors make our $\mathrm{K}_{\mathrm{IR}}$ current model an approximation to this current in accumbal cells.

The Hodgkin-Huxley parameters for modeling the $\mathrm{K}_{\mathrm{A}}$ channel were based on those used by Traub [21] for hippocampal pyramidal cells, and modified to fit the data reported by Gabel and Nisenbaum [22] in rat neostriatum. As with the $\mathrm{K}_{\mathrm{IR}}$ current, we explored the effects of small shifts in the $\mathrm{K}_{\mathrm{A}} I-V$ curve on the bistability of the membrane potential. These potassium currents serve as the anchors for the two membrane states. Transition between states depends upon driving the potential past the crossing-point of the curves. In our studies, we found that one of the critical elements for attaining bistability is that the $\mathrm{K}_{\mathrm{IR}}$ channel 




Voltage (mV)

Fig. 3. $I-V$ curve for the $\mathrm{K}_{\mathrm{IR}}$ channel.

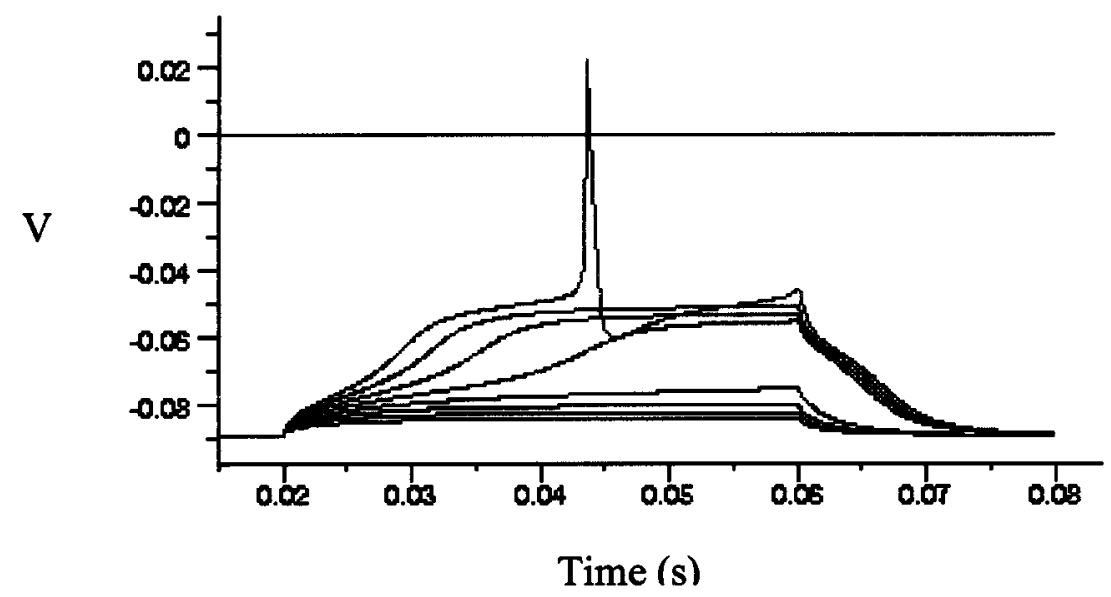

Fig. 4. Bistability of membrane potential. Voltage induced by a series of graded current injections from 1 to $10 \mathrm{nA}$. Note that the membrane potential tends to remain near either the $\mathrm{K}_{\mathrm{IR}}$ or $\mathrm{K}_{\mathrm{A}}$ reversal potentials, which define the down and up states.

close at a slightly more hyperpolarized voltage than that at which the $\mathrm{K}_{\mathrm{A}}$ channel begins to substantially open.

\section{B. Membrane Mechanisms Underlying the Two-State Potential}

Incorporating the $\mathrm{K}_{\mathrm{IR}}$ and $\mathrm{K}_{\mathrm{A}}$ currents into our 29-compartment MSP neuron model shows that these two currents (along with the standard $\mathrm{Na}^{+}$and $\mathrm{K}_{\mathrm{DR}}$ currents), are sufficient to generate the two-state, bistable membrane potential. Fig. 4 shows the results of a series of ten current injections applied to the soma of the modeled MSP neuron. The current injections were graduated from 0.2 to $0.5 \mathrm{nA}$ in equal increments, each lasting $40 \mathrm{~ms}$. Increasing currents lead to greater depolarization, but there is a clear nonlinearity in the steady-state voltage level reached, with a bifurcation into an "up" and "down" state. The highest level of current injection leads to the generation of action potentials from the "up" state. These results closely correspond to recordings from accumbal cells, e.g., Fig. 1 from Lopes da Silva et al. [23].
In the down state, small current injections are countered by the $\mathrm{K}_{\mathrm{IR}}$ current, which acts to keep the potential near the reversal potential of $-90 \mathrm{mV}$. Larger injections are able to depolarize the membrane, and begin to close $\mathrm{K}_{\mathrm{IR}}$. As Wilson has pointed out [4], closing the $\mathrm{K}_{\mathrm{IR}}$ channels increases membrane resistance, thus increasing current generates a nonlinear increase in depolarization. This nonlinearity helps propel the potential from the "down" state to the "up" state. However, sufficient depolarization begins to open $\mathrm{K}_{\mathrm{A}}$ channels, which counter the injected currents, and tend to stabilize the potential near $-60 \mathrm{mV}$. Increasing depolarization opens more $\mathrm{K}_{\mathrm{A}}$ channels, decreasing membrane resistance, and thus reducing the induced potential change. A disproportionate increase in the injected current is needed to depolarize the cells the last few millivolts above $-60 \mathrm{mV}$ required to generate action potentials. The two potassium channels, with their offset and opposing voltage dependencies, thus create a kind of "energy barrier" to depolarizing the membrane potential. This barrier defines 


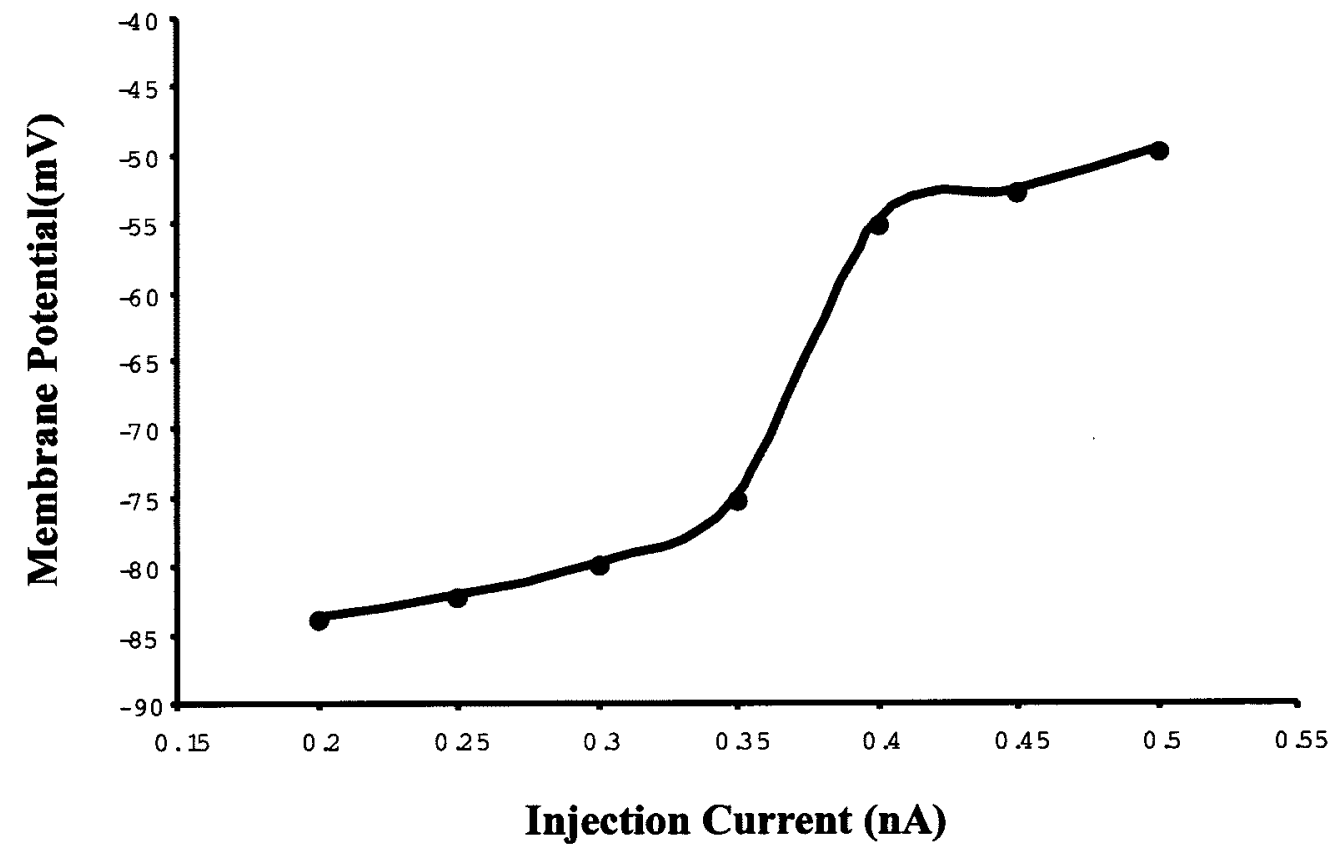

Fig. 5. Depolarization as a function of synaptic Drive. Steady-state membrane potential achieved with increasing amplitudes of current injection to the soma. Note the nonlinear change in membrane potential.

the two membrane potential states. Note however, that continued current injection is required to keep the membrane in the "up" state; when the current is terminated, the potential quickly falls back to the "down" state (Fig. 4).

Wilson [4] has stressed that the bistable membrane potential is not an intrinsic membrane property, but rather, ongoing synaptic input is required to maintain the cell in the up state. In the model used here, $\mathrm{K}_{\mathrm{IR}}$ and $\mathrm{K}_{\mathrm{A}}$ act to maintain the potentials that define the down and up states, respectively. However, the membrane potential also reflects the input synaptic current, and thus assumes a continuum of values. It is important to note that MSP neurons contain a number of other intrinsic conductances, many or all of which may contribute to the stability of the membrane states. We do not claim that $\mathrm{K}_{\mathrm{IR}}$ and $\mathrm{K}_{\mathrm{A}}$ solely determine the bistability, rather, that alone they are sufficient to provide a major component of bistability.

In vivo, the striking nature of the bistable states is their persistence over $500 \mathrm{~ms}$ or longer (see Fig. 2), and we therefore wondered whether longer lasting currents, such as L-type $\mathrm{Ca}^{2+}$ channels and/or NMDA receptor currents might allow the cell to persist in the up state in the absence of synaptic input. We stimulated the cell with a synchronized barrage of synaptic inputs - the inputs arrived within a 5-ms time window, and barrages were delivered at $30 \mathrm{~Hz}$. This stimulation frequency was chosen to correspond to a typical firing rate of delay-period neurons in prefrontal cortex (see discussion). However, our studies revealed that even in the presence of these additional currents, the cell was not able to remain in the up state. Activation of NMDA currents should, in principle, be able to maintain the up state; however, the voltage in the up state is not sufficiently depolarized to release NMDA receptors from the $\mathrm{Mg}^{2+}$ block.
The integrative properties of the cell determine the spatial and temporal constraints required of the input stream, as well as the number of inputs required to depolarize the cell into the up state. Fig. 5 shows the steady-state membrane potential achieved for different levels of current injection. At roughly $0.4 \mathrm{nA}$, there is a nonlinear increase in the steady-state potential achieved; this corresponds to a shift to the up state.

We also studied the effect of applying a synchronized barrage of AMPA-mediated synaptic inputs to the cell. Inputs were delivered in a barrage to the most proximal compartment on a single dendrite, with spike times distributed in a Gaussian distribution. Fig. 6 shows the number of inputs required to switch the cell into the up state, as a function of the width of the Gaussian. Plotted on the abcissa is the standard deviation of the Gaussian, in milliseconds, i.e., roughly the time interval over which $63 \%$ of the inputs arrived. The three curves correspond to different densities of $\mathrm{K}_{\mathrm{IR}}$ conductance. Increase in the $\mathrm{K}_{\mathrm{IR}}$ current, such as would occur in the presence of dopamine (see below), increases the number or temporal synchrony of inputs required to switch the cell to the up state. The data shows that on the order of 100-450 roughly synchronous inputs are required for the transition. This number can be compared to the estimate speculated by Lopes da Silva et al. [16], who suggested an order of magnitude less inputs would be sufficient. Our findings reflect the effects of the $K_{I R}$ channel as well as some aspects of dendritic geometry. The required number of inputs increases roughly linearly with the temporal dispersion, but with a small slope, so that the density of $\mathrm{K}_{\mathrm{IR}}$ current is the major factor in determining how many inputs are required.

\section{Effects of Dopamine on Cell Function}

The modulatory effects of dopamine arise from effects on a range of membrane channels together with effects on both 


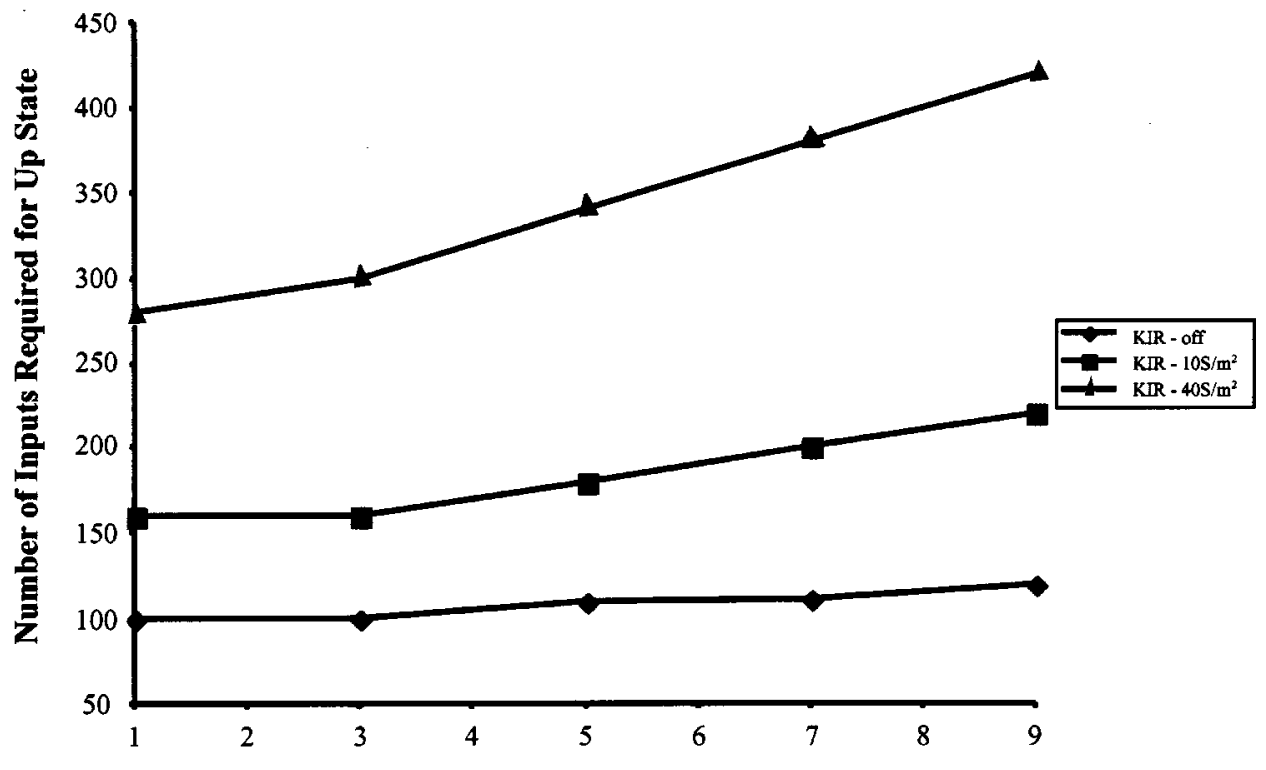

Temporal Dispersion of Synaptic Inputs (ms)

Fig. 6. Number of synchronized inputs required to switch to the up state. Barrages of AMPA synaptic inputs were delivered to the MSP cell, timing of spikes in the barrage were Gaussian distributed, with a $\sigma$ shown on the abcissa. The greater the temporal dispersion, the greater number of inputs is required to switch to the up state. The three curves show the effect of increasing the number of $\mathrm{K}_{\mathrm{IR}}$ channels in the dendrites, and demonstrate that the magnitude of $\mathrm{K}_{\mathrm{IR}}$ is a major factor in controlling the ability to switch states. Note that a state transition requires between $100-450$ inputs.

glutamatergic and GABAergic synaptic transmission [11]. A variety of subtypes of dopamine receptors (D1-D5) are expressed on different cell types. ${ }^{4}$ However, all dopamine receptors act via $\mathrm{G}$ protein signaling to modulate adenylyl cyclase: D1 receptors activate adenylyl cyclase, whereas D2 and $\mathrm{D} 3$ receptors inhibit adenylyl cyclase [11]. By increasing adenylyl cyclase, D1 agonists decrease the peak $\mathrm{Na}^{+}$current and both $\mathrm{N}$ and $\mathrm{P} / \mathrm{Q} \mathrm{Ca}^{2+}$ currents, and increase both $\mathrm{K}_{\mathrm{IR}}$ and L-type $\mathrm{Ca}^{2+}$ currents.

In cellular recordings, dopamine has been found to be capable of either exciting or inhibiting MSP activity. These differences may arise from the receptor subtypes activated. However, even studies from the same lab activating D1 and D2 receptors (some carried out in D3 knock-out mice) show conflicting results [24], [25]. Nicola et al. [11] suggest that the reason for these conflicting findings is that the effect of dopamine depends on the state (up versus down) of the MSP neuron. They propose that in the down state, D1 activation suppresses excitation by depressing the $\mathrm{Na}^{+}$current and enhancing the $\mathrm{K}_{\mathrm{IR}}$ current. For cells in the up state, where $\mathrm{K}_{\mathrm{IR}}$ is deactivated, D1 input increases the L-type $\mathrm{Ca}^{2+}$ current and NMDA currents (but suppresses AMPA currents). D1 activation is thought to stabilize neurons in their current state and to increase the barrier to switching states [11]. This is supported by in vivo experiments showing that dopamine suppresses background activity while enhancing goal-directed activity [26].

\footnotetext{
${ }^{4}$ The modern era of schizophrenia therapy began with the discovery that the clinical efficacy of various antipsychotic agents was exactly correlated with their binding affinity to the D2 subtype of dopamine receptor. Newer drugs target D3 receptors, which are selectively expressed in the limbic areas of cortex, including Nacc.
}

We modeled the effects of dopamine on our MSP neuron by doubling $\mathrm{K}_{\mathrm{IR}}$ from its baseline value of $50 \mathrm{~S} / \mathrm{m}^{2}$ and quadrupling the L-type $\mathrm{Ca}^{2+}$ conductance from its baseline value of $5 \mathrm{~S} / \mathrm{m}^{2}$ [27]. These baseline conductances correspond to the low-end values reported in the literature, and the increase due to dopaminergic stimulation corresponds to the high-end values observed experimentally [27], [28]. The L-type $\mathrm{Ca}^{2+}$ channel was constructed based on data reported for the high-voltage activated channel [27]. Dopaminergic effects on AMPA conductances were not included. To investigate the up state, the cell was injected with $0.6 \mathrm{nA}$ current for $50 \mathrm{~ms}$. Separate simulations show that this current corresponds to approximately 6000 synaptic inputs per second. To investigate the down state, the cell was stimulated with a barrage of 500 synaptic inputs per second, and an additional barrage of 100 inputs delivered over a 10-ms interval, as shown in Fig. 7.

As shown in Fig. 7, the effect of dopamine on the MSP cell depends on cell state. In the down state, the response to the additional input (over background) is suppressed by dopamine [compare Fig. 7(d) to (c)]. However, in the up state, dopamine causes a marked increase in firing frequency in response to this same synaptic input. These results correspond to those obtained experimentally by Hernandez-Lopez et al. [29], who showed that dopamine applied to the up state decreases the interspike interval of the firing cell. Note that even though dopamine acts on both the $\mathrm{K}_{\mathrm{IR}}$ and the $\mathrm{Ca}^{2+}$ current, since each current is only active in one of the states (L-type $\mathrm{Ca}^{2+}$ in the up state, $\mathrm{K}_{\mathrm{IR}}$ in the down state), the net effect of dopamine is state-dependent. Increased $\mathrm{K}_{\mathrm{IR}}$ is responsible for suppressing the effect to synaptic input in the down state, and increased $\mathrm{Ca}^{2+}$ current 

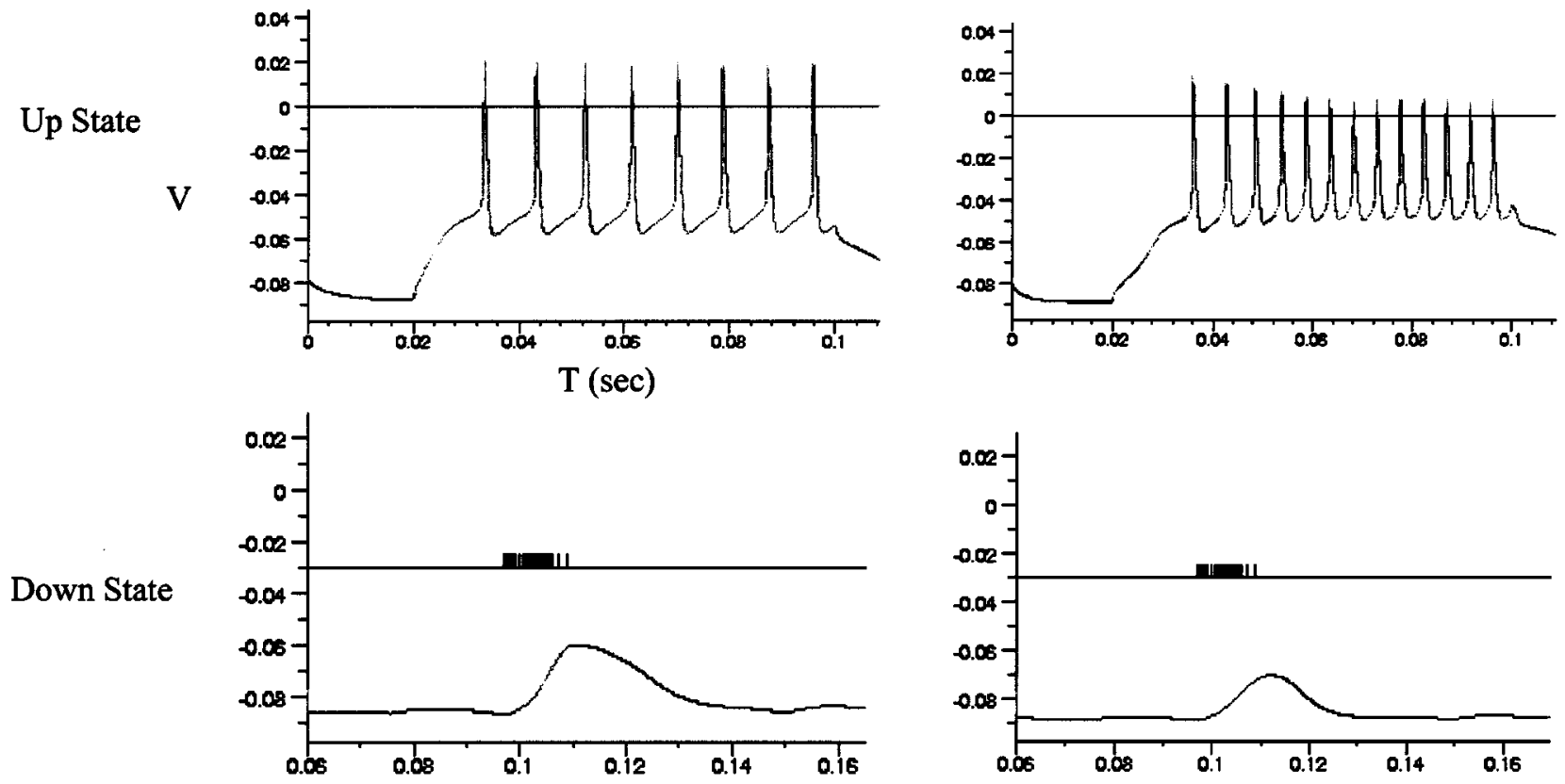

Fig. 7. Effect of dopamine on MSP model neuron response. (Top) Cells were injected with 0.6 $\mathrm{nA}$ for $50 \mathrm{~ms}$, which led to repeated cell firing. Dopamine causes an increase in the frequency of firing. (Bottom) Cells were stimulated with a background input of 500 spikes per second, an additional barrage of 100 synaptic inputs was applied distributed according to a Gaussian distribution with a 5-ms standard deviation. In the down state, the effect of dopamine is to suppress the response to the synchronized inputs.

leads to greater excitability in the up state. We have not incorporated dopaminergic effects on synaptic conductances, which might further increase the observed effect, and may compensate for the accommodation in spike amplitudes seen in Fig. 7(b).

\section{DISCUSSION}

The MSP neuron model developed here represents an initial step toward a model of the Nacc, and insight into the role of the accumbens in cognitive integration must await network simulations. Nevertheless, this initial model sheds some light on the four questions posed above, and leads to some speculative hypotheses.

The model demonstrates that the bistable membrane potential is likely mediated, in large part, by the interplay between two voltage-dependent potassium currents, $\mathrm{K}_{\mathrm{IR}}$ and $\mathrm{K}_{\mathrm{A}}$. The nonlinear transition between states requires that with increasing depolarization, $\mathrm{K}_{\mathrm{IR}}$ closes before $\mathrm{K}_{\mathrm{A}}$ opens. We used identical maximal conductance values for these currents $\left(100 \mathrm{~S} / \mathrm{m}^{2}\right)$, so that membrane resistance is roughly equivalent in the up and down state. Monitoring total membrane conductance as the cell undergoes state transitions (data not shown) indicates that the membrane time constant decreases with depolarization, leveling out as it approaches the up state. This is in agreement with previous modeling results reported by Wilson [5].

We were unable to maintain the cell in the up state in the absence of continued stimulation. Thus, in agreement with
Wilson [4], we find that the up state is not an intrinsically stable membrane state, but requires ongoing stimulation. Of particular interest is whether PFC inputs, on their own, are capable of maintaining the MSP in the up state. We found this not to be the case: stimulation with a sufficient number of synchronized inputs delivered at $30-\mathrm{Hz}$ switches the cell to the up state, but the potential falls back to the down state before the next input barrage (33 ms). Theoretically, it should be possible for long-lasting currents from NMDA receptors or $\mathrm{Ca}^{2+}$ channels to maintain the up state. However, in our simulations, the up state voltage $(\sim-60 \mathrm{mV})$ was insufficient to maintain NMDA activation, and the L-type $\mathrm{Ca}^{2+}$ current implemented here was similarly unable to generate sufficient current to maintain the up state. Maintenance of the up state, therefore, appears to require ongoing input to the cell. However, it remains to be determined whether network properties among coupled MSP cells might contribute to the persistence of the up state.

Our investigation of the effects of dopamine on cell response are in agreement with the evidence put forward by Malenka et al. [11] and with recent modeling studies in prefrontal cortex [30]. We found that dopamine acts to stabilize cells in their present state, or to increase the barrier to state transitions. In the down state, dopamine accentuates the $\mathrm{K}_{\mathrm{IR}}$ current, damping out the effects of afferent stimulation; in the up state, dopamine accentuates the L-type $\mathrm{Ca}^{2+}$ current, which amplifies cell responses to input. We have only incorporated D1 receptor effects, and thus the effect could be further dissociated according to synaptic location or cell type. 
The ability of dopamine to change cell gain in a state-dependent manner allows the cell to respond in a context-dependent manner.

\section{A. What Controls the Membrane State}

Given our conclusion that continuous inputs are required to maintain the cell in the up state, the question arises of which afferent sources provide those inputs: PFC, hippocampus, and/or amygdala. Published studies provide some insight into the firing characteristics of the outputs of these structures, as well as their functional roles.

Prefrontal Input: Dorsolateral prefrontal cortex is an area critical for working memory, high-level problem solving, and planning, and it is centrally implicated in the symptomatology of schizophrenia [31]. Cells in prefrontal cortex show sustained activation during working memory tasks, such as delayed-nonmatch-to-sample [32]. In such tasks, for example, a pebble might be placed under one of two identical boxes on a table, the animal must maintain in memory the location of that box, and after a delay period (e.g., $30 \mathrm{~s}$ ) reach for the box which does not contain the pebble. Fuster [32] and others have shown that during the "delay" period, prefrontal cortex cells fire at a relatively high rate $(\sim 30 \mathrm{~Hz})$, and cease firing as the motor portion (reaching for the box) of the task begins. Prefrontal cortex is considered to be the highest integrative center linking perception and action. Delay period activity is found in several cortical areas, but cells in other cortical areas (e.g., inferotemporal cortex) switch firing patterns to each new visual stimulus introduced-PFC cells maintain "goalrelated" activity regardless of intervening stimuli [33].

Durstewitz et al. [30] have developed a biophysical-level model of a PFC network composed of layer V pyramidal cells and fast spiking interneurons. The behavior of this network bears a number of similarities to the MSP cells considered here. The pyramidal cells exhibit two activity states: a "down" state in which spontaneous firing occurs at $\sim 1-3$ $\mathrm{Hz}$, and an "up" state, corresponding to the more vigorous delay-period activity with $\sim 30-\mathrm{Hz}$ firing. Durstewitz et al. incorporated the effects of dopaminergic modulation by appropriate shifts in channel conductances. Their results show that dopamine can have either an excitatory or a suppressive effect-depending upon the firing state of the cell. When the network is in the high-activity delay-period state, the net effect of dopamine is to sustain cells with high-activity and to suppress cells that are not firing. In the low-activity state, the suppressive effects of dopamine predominate (due to increased GABAergic and reduced AMPA conductance). Thus, dopamine acts to enhance goal-related activity, and to insulate these activity states from perturbations due to ongoing inputs to PFC.

Hippocampal Input: The accumbens receives a massive projection from several areas of the hippocampal formation, including area CA1, subiculum, and entorhinal cortex. Functional mapping studies show intense activation of CA1 and the subiculum during working memory and associative memory tasks [34].
Estimates can be made of the spatiotemporal patterns of activity impinging on Nacc from the hippocampus. In rodents, the activity of "place" cells in CA1 corresponds to the animal's location in a known environment. During a spatial navigation task, such as navigating through a maze toward a food reward, the firing pattern in CA1 corresponds to successively activated constellations of place cells. Under such experimental conditions, statistical decoding of spike train data demonstrates that spatial position can be reliably encoded in the spiking patterns of as little as 30 neurons [35]. The degree of convergence of these place cells onto single MSP neurons is not known; however, as the animal moves through the receptive field of a place cell, there is a precession in the timing of spike firing relative to the phase of the underlying theta rhythm (a slow 6-10 Hz synchronous modulation of firing across CA1). Also, there is a linear increase in the firing rate proportional to the speed at which the animal is running [36]. At customary speeds of rodent movement $(10-20 \mathrm{~cm} / \mathrm{s})$, the animal spends several theta cycles (100-167 ms) traversing one place cell receptive field $(\sim 10$ $\mathrm{cm})$. Interestingly, dopamine also modulates cell properties and interactions within the hippocampus [37].

\section{B. What Does the Accumbens Compute?}

What might be the effect on a network of accumbal cells of the converging afferent inputs? MSP cells mutually inhibit each other via GABAergic synapses; however, they also share mutual excitation via gap junctions. Connors et $a l$. have shown that such a network architecture is capable of rapid synchronization [38]. They investigated low-threshold spiking interneurons in neocortex, which are interconnected by both GABAergic synapses and gap junctions, and showed that such networks could be synchronized by the application of neuromodulators, such as acetylcholine. And Hopfield [39] has shown that in such networks, the transient synchrony emerging and re-emerging over time among different subpopulations of neurons can carry out interesting computations, including recognition and generalization. It is interesting to speculate that dopamine might serve to transiently synchronize accumbal networks. Grace et al. [12] have evidence that gap junctional coupling between MSP cells is controlled by NO (nitric oxide) released in response to dopamine. And in several systems, including the retina, dopamine has been shown to change the gain of a local network via modulation of gap junction conductances. Thus, selective modulation by dopamine might lead to the emergence of transiently synchronized MSP cell assemblies, which act as temporary routers on the reentrant feedback loop to prefrontal cortex. Since the release of dopamine by the tegmentum is controlled by the cortex, this complex reentrant circuit is capable of self-modulating the computations carried out on the basis of the combined afferent input to accumbens. A number of models have made use of this interplay between feedback and neuromodulation to account for the role of prefrontal cortex in executive volitional control [40]-[42]. 


\section{Functional Role of Pattern Integration}

One possible conception of the computation performed by the accumbens, couched in terms of spatial navigation, is as follows. PFC generates a vigorous activity pattern corresponding to the goal location. Hippocampal afferents will convey the sequence of place cell activations-either experienced or imagined. Both of these inputs will impinge upon the Nacc network, and based on the current position and the goal position, the Nacc will predict whether the trajectory is directed toward the goal. This prediction will be expressed as the pattern of MSP cells with synchronized firing.

This process of pattern integration might be carried out by associative memory mechanisms that learn to associate patterns across the input streams. A second class of possible mechanisms however would allow the accumbens to compute a prediction of whether the recent inputs received via the hippocampal channel are progressing toward the goal defined by the PFC channel. Such a network would more closely resemble a Kalman filter, and the implementation might resemble that suggested for predictive coding in the cortico-thalamic loop [43]. Interestingly, there is recent evidence suggests that schizophrenics are impaired in predicting trajectories [44].

\section{Application to Understanding Schizophrenia}

It would be premature to extrapolate these simple modeling studies to the pathophysiology of schizophrenia; however, it is intriguing to consider how future work might move toward that goal. One approach is to introduce the types of pathology found in schizophrenic brains, and to observe the consequences on network function. Recent work by Benes [45] points to a loss of GABAergic interneurons and concomitant changes in $\mathrm{GABA}_{\mathrm{A}}$ receptor densities in CA3 and CA1 of schizophrenic brains. Benes proposes that in $\mathrm{CA} 3$, the major loss is in disinhibitory interneurons, leading to $\mathrm{GABA}_{\mathrm{A}}$ upregulation on other interneurons, whereas in CA1, loss of inhibitory interneurons leads to $\mathrm{GABA}_{\mathrm{A}}$ upregulation on pyramidal cells.

Several lines of recent evidence suggest that schizophrenia may arise from developmental abnormalities in the hippocampus, and that this abnormal hippocampal input to Nacc affects PFC-accumbens interactions in adult life. This provides an interesting parallel to Alzheimer's disease, in which pathological changes originate near the hippocampal formation and gradually spread outward to successively connected regions [46].

In the prefrontal cortex, layer $\mathrm{V}$ pyramidal cells are the source of the projection to Nacc; however, the strongest evidence of PFC pathology in schizophrenia is in layer III. Lewis et al. [47] find decreased pyramidal cell soma sizes, decreased densities of chandelier-type interneurons, and a decrease or disorganization of long-range excitatory horizontal connections between layer III pyramidal cells. These long-range horizontal connections mature at adolescence, just at the time when schizophrenic symptoms are manifested. In previous modeling studies, it has been demonstrated that long-range connections are capable of synchronizing the firing of cortical cells, particularly in the presence of appropriate neuromodulators [48]. These findings, together with the evidence reviewed by Lopes da Silva et al. [16] suggest that schizophrenia may arise from a fundamental defect in the mechanisms controlling transient synchronization in Nacc, with functional consequences at multiple levels for its afferent and efferent partners.

\section{ACKNOWLEDGMENT}

The authors would like to thank $\mathrm{C}$. Wilson for providing the code for his potassium channel models as well as a number of insightful and helpful suggestions, and E. Menschik for guidance on the implementation.

\section{REFERENCES}

[1] L. Heimer, "Basal forebrain in the context of schizophrenia," Brain Res., Brain Res. Rev., vol. 31, pp. 205-305, 2000.

[2] H. Beckmann and M. Lauer, "The human striatum in schizophrenia. II. Increased number of striatal neurons in schizophrenics," Psych. Res., vol. 68, pp. 99-109, 1997.

[3] L. Finkel, "Neuroengineering models of brain disease," Annu. Rev. Biomed. Eng., vol. 2, pp. 577-606, 2000.

[4] C. J. Wilson and Y. Kawaguchi, "The origins of two-state spontaneous membrane potential fluctuations of neostriatal spiny neurons," J. Neurosci., vol. 16, pp. 2397-2410, 1996.

[5] C. Wilson, "Dendritic morphology, inward rectification, and the functional properties of neostriatal neurons," in Single Neuron Computation, T. McKenna, J. Davis, and S. F. Zornetzer, Eds. Boston, MA: Academic, 1992, pp. 141-172.

[6] — , "The generation of natural firing patterns in neostriatal neurons," Prog. Brain Res., vol. 99, pp. 277-297, 1993.

[7] E. A. Stern, D. Jaeger, and C. J. Wilson, "Membrane potential synchrony of simultaneously recorded striatal spiny neurons in vivo," Nature, vol. 394, pp. 475-478, 1998.

[8] D. R. Weinberger, "Implications of normal brain development for the pathogenesis of schizophrenia," Arch. Gen. Psych., vol. 44, pp. 660-669, 1987.

[9] M. Karreman and B. Moghaddam, "The prefrontal cortex regulates the basal release of dopamine in the limbic striatum: An effect mediated by ventral tegmental area," J. Neurochem., vol. 66, pp. 589-598, 1996.

[10] M. T. Taber and H. C. Fibiger, "Electrical stimulation of the prefrontal cortex increases dopamine release in the nucleus accumbens of the rat: Modulation by metabotropic glutamate receptors," J. Neurosci., vol. 15, pp. 3896-3904, 1995.

[11] S. M. Nicola, J. Surmeier, and R. C. Malenka, "Dopaminergic modulation of neuronal excitability in the striatum and nucleus accumbens," Annu. Rev. Neurosci., vol. 23, pp. 185-215, 2000.

[12] P. O'Donnell and A. A. Grace, "Dysfunctions in multiple interrelated systems as the neurobiological bases of schizophrenic symptom clusters," Schizophr. Bull., vol. 24, pp. 267-283, 1998.

[13] - "Synaptic interactions among excitatory afferents to nucleus accumbens neurons: Hippocampal gating of prefrontal cortical input," J. Neurosci., vol. 15, pp. 3622-3639, 1995.

[14] M. Koch, "The neurobiology of startle," Prog. Neurobiol., vol. 59, pp. $107-128,1999$.

[15] F. J. Wan and N. R. Swerdlow, "Sensorimotor gating in rats is regulated by different dopamine-glutamate interactions in the nucleus accumbens core and shell subregions," Brain Res., vol. 722, pp. 168-1776, 1996.

[16] C. M. Pennartz, H. J. Groenewegen, and F. H. Lopes da Silva, "The nucleus accumbens as a complex of functionally distinct neuronal ensembles: An integration of behavioral, electrophysiological and anatomical data," Prog. Neurobiol., vol. 42, pp. 719-761, 1994.

[17] J. Bower and D. Beeman, The Book of GENESIS. New York: Springer/Telos, 1998.

[18] C. M. A. Pennartz, M. J. Dolleman-Van der Weel, and Lopes da Silva, "Differential membrane properties and dopamine effects in the shell and core of the rat nucleus accumbens studied in vitro," Neurosci. Lett., vol. 136, pp. 109-112, 1992. 
[19] H. Hayashi and H. M. Fishman, "Inward rectifier K-channel kinetics from analysis of the complex conductance of Aplysia neuronal membrane," Biophys. J., vol. 53, pp. 747-757.

[20] S. Hagiwara and K. Takahashi, "The anamolous rectification and cation selectivity of a starfish egg cell," J. Membr. Biol., vol. 18, pp. 61-80, 1974.

[21] R. D. Traub, J. G. Jefferys, R. Miles, M. A. Whittington, and K. Toth, "A branching dendritic model of a rodent CA3 pyramidal neurone," J. Physiol. (Lond.), vol. 481, pp. 79-95, 1994.

[22] L. A. Gabel and E. S. Nisenbaum, "Biophysical characterization and functional consequences of a slowly inactivating potassium current in neostriatal neurons," J. Neurophysiol., vol. 79, pp. 1989-2002, 1998.

[23] C. M. A. Pennartz, P. H. Boeijinga, S. T. Kitai, and F. H. Lopes da Silva, "Contribution of NMDA receptors to postsynaptic potentials and paired-pulse facilitation in identified neurons of the rat nucleus accumbens in vitro," Exp. Brain Res., vol. 86, pp. 190-198, 1991.

[24] X. Hu and F. White, "Dopamine enhances glutamate-induced excitation of rat striatal neurons by cooperative activation of D1 and D2 class receptors," Neurosci. Lett., vol. 224, pp. 61-65, 1997.

[25] — , "Loss of D1/D2 dopamine receptor synergisms following repeated administration of D1 or D2 receptor selective antagonists: Electrophysiological and behavioral studies," Synapse, pp. 43-61, 17.

[26] E. A. Kiyatkin and G. V. Rebec, "Striatal neuronal activity and responsiveness to dopamine and glutamate after selective blockade of D1 and D2 dopamine receptors in freely moving rats," J. Neurosci., vol. 19, pp. 3594-3609, 1999.

[27] R. B. Avery and D. Johnston, "Multiple channel types contribute to the low-voltage-activated calcium current in hippocampal CA3 pyramidal neurons," J. Neurosci., vol. 16, pp. 5567-5582, 1996.

[28] J. Bargas, A. Howe, J. Eberwine, Y. Cao, and D. J. Surmeier, "Cellular and molecular characterization of Ca currents in acutely isolated adult rat neostriatal cells," J. Neurosci., vol. 14, pp. 6667-6686, 1994.

[29] S. Hernandez-Lopez, J. Bargas, D. J. Surmeier, A. Reyes, and E. Galarraga, "D1 receptor activation enhances evoked discharge in neostriatal medium spiny neurons by modulating an L-type $\mathrm{Ca}^{2+}$ conductance," J. Neurosci., vol. 17, pp. 3334-3342, 1997.

[30] D. Durstewitz, M. Kelc, and O. Gunturkun, "A neurocomputational theory of the dopaminergic modulation of working memory functions," J. Neurosci., vol. 19, pp. 2807-2822, 1999.

[31] P. S. Goldman-Rakic, "The physiological approach: Functional architecture of working memory and disordered cognition in schizophrenia," Biol. Psych., vol. 46, pp. 650-661, 1999.

[32] J. M. Fuster, "Memory networks in the prefrontal cortex," Prog. Brain Res., vol. 122, pp. 309-316.

[33] L. Chelazzi, J. Duncan, E. K. Miller, and R. Desimone, "Responses of neurons in inferior temporal cortex during memory-guided visual search," J. Neurophysiol., vol. 80, pp. 2918-2940, 1998.

[34] E. Sybirska, L. Davachi, and P. S. Goldman-Rakic, "Prominence of direct entorhinal-CA1 pathway activation in sensorimotor and cognitive tasks revealed by 2 -DG functional mapping in nonhuman primate," J. Neurosci., vol. 20, pp. 5827-5834, 2000.

[35] E. N. Brown, L. M. Frank, D. Tang, M. C. Quirk, and M. A. Wilson, "A statistical paradigm for neural spike train decoding applied to position prediction from ensemble firing patterns of rat hippocampal place cells," J. Neurosci., vol. 18, pp. 7411-7425, 1998.

[36] K. Zhang, I. Ginzburg, B. L. McNaughton, and T. J. Sejnowski, "Interpreting neuronal population activity by reconstruction: Unified framework with application to hippocampal place cells," J. Neurophysiol., vol. 79, pp. 1017-1044, 1998.
[37] N. A. Otmakhova and J. E. Lisman, "Dopamine selectively inhibits the direct cortical pathway to the CA1 hippocampal region," J. Neurosci., vol. 19, pp. 1437-1445, 1999.

[38] K. Zhang, M. Beierlein, J. R. Gibson, and B. W. Connors, "A network of electrically coupled interneurons drives synchronized inhibition in neocortex," Nat. Neurosci., vol. 3, pp. 904-910, 2000.

[39] J. J. Hopfield and C. D. Brody, "What is a moment? "Cortical" sensory integration over a brief interval," in Proc. Natl. Acad. Sci., vol. 97, USA, 2000, pp. 13 919-13924.

[40] T. S. Braver, D. M. Barch, and J. D. Cohen, "Cognition and control in schizophrenia: A computational model of dopamine and prefrontal function," Biol. Psych., vol. 46, pp. 312-328, 1999.

[41] S. Dehaene and J. P. Changeux, "Neuronal models of prefrontal cortical functions," Ann. NY Acad. Sci., vol. 769, pp. 305-319, 1995.

[42] A. Compte, N. Brunel, P. S. Goldman-Rakic, and X. J. Wang, "Synaptic mechanisms and network dynamics underlying spatial working memory in a cortical network model," Cereb. Cortex, vol. 10, pp. 910-923, 2000.

[43] E. Ahissar, R. Sosnik, and S. Haidarliu, "Transformation from temporal to rate coding in a somatosensory thalamocortical pathway," Nature, vol. 406, pp. 302-306, 2000.

[44] C. Hooker and S. Park, "Trajectory estimation in schizophrenia," Schizophr. Res., vol. 45, pp. 83-92, 2000.

[45] F. M. Benes, "Emerging principles of altered neural circuitry in schizophrenia," Brain Res. Rev., vol. 31, pp. 251-269, 2000.

[46] M. E. Hasselmo, "A computational model of the progression of Alzheimer's disease," MD Comput., vol. 14, pp. 181-191, May-June 1997.

[47] D. A. Lewis and G. Gonzalez-Burgos, "Intrinsic excitatory connections in the prefrontal cortex and the pathophysiology of schizophrenia," Brain Res. Bull., vol. 52, pp. 309-317, 2000.

[48] S.-C. Yen, E. D. Menschik, and L. H. Finkel, "Perceptual grouping in striate cortical networks mediated by synchronization and desynchronization," Neurocomputing, vol. 26-27, pp. 609-616, 1999.

[49] P. O'Donnell, J. Greene, N. Pabello, B. Lewis, and A. A. Grace, "Modulation of cell firing in the nucleus accumbens," Ann. NY Acad. Sci., vol. 8877, pp. 157-175, 1999.

John A. Wolf received the B.A. degree in biology from the University of Pennsylvania, Philadelphia, in 1994. He is currently a Ph.D. student in neuroscience there.

He has previously worked in the area of CNS trauma, where he is an author of more than ten publications. His research interests are in neurophysiological analysis and computational modeling of accumbal interactions.

Lee F. Schroeder received the B.A. degree in physics from the University of Pennsylvania, Philadelphia, in 1996. He has worked on functional imaging in the Neuropsychiatry division there. His current interests are in computational neuroscience, which he will pursue as an M.D./Ph.D. student.

Leif H. Finkel received the Ph.D. and M.D. degrees from the University of Pennsylvania, Philadelphia.

He is currently Professor of Bioengineering at the University of Pennsylvania. His research interests include computational models of visual perception as well as neuroengineering simulations of neurological and psychiatric disease. 